\title{
Research on Image Reconstruction Algorithms for Tuber Electrical Resistance Tomography System
}

\author{
Zili Jiang , Xiang Deng, Shuyi Liu and Yu Tang \\ School of Mechanical, Electronic and Control Engineering, Beijing Jiaotong University, Beijing.100044, P.R.China
}

\begin{abstract}
The application of electrical resistance tomography (ERT) technology has been expanded to the field of agriculture, and the concept of TERT (Tuber Electrical Resistance Tomography) is proposed. On the basis of the research on the forward and the inverse problems of the TERT system, a hybrid algorithm based on genetic algorithm is proposed, which can be used in TERT system to monitor the growth status of the plant tubers. The image reconstruction of TERT system is different from the conventional ERT system for two phase-flow measurement. Imaging of TERT needs more precision measurement and the conventional ERT cares more about the image reconstruction speed. A variety of algorithms are analyzed and optimized for the purpose of making them suitable for TERT system. For example: linear back projection, modified Newton-Raphson and genetic algorithm. Experimental results showed that the novel hybrid algorithm is superior to other algorithm and it can effectively improve the image reconstruction quality.
\end{abstract}

\section{Introduction}

Precision agriculture is a new trend of modern agricultural development in the world, and it is the system driven by information technology and a full set of modern agricultural management and production means with the locating, timely, quantifiably and the spatial variation characteristics ${ }^{[1]}$. At present, the research of electrical resistance tomography (ERT) technology is mainly focused on the industrial multiphase-flow field. In the paper, the application of ERT technology in the agriculture is described and the concept of TERT (Tuber Electrical Resistance Tomography) is proposed. TERT is a new research field. By the TERT system, the growth morphology of plant tubers and soil environment of the plant growth can be better monitored timely.

Image reconstruction of TERT system is a key problem and the research result can provide powerful tool for the visualization monitoring of the growth process characteristics of plant tubers such as precious and Chinese herbal medicines ginseng, etc. Therefore, it is very meaningful to study the image reconstruction algorithms of plant tubers tomography.

In order to meet the accuracy and real-time requirements of the application in ERT system, a variety of image reconstruction algorithms ${ }^{[2][3][4]}$ had been proposed in the past. In this paper, on the research of TERT Image reconstruction algorithms, the algorithms such as linear back projection algorithm ${ }^{[5]}$ (LBP), Newton-Raphson algorithm ${ }^{[6]}$ (MNR) and genetic algorithm $^{[7]}$ (GA), applied to the TERT system, are systematically studied and optimized under different conditions.

The LBP algorithm is the simplest and most rough image reconstruction algorithm. Although it has the advantages of simple and fast, the experiments show that it has better reconstruction effect only when the actual resistivity distribution is homogeneous. MNR algorithm is a kind of iterative reconstruction algorithm with a perfect and precise characteristics and better practical application, but it is not ideal in most cases because of the ill conditioned Hessian matrix. In some related literature, the genetic algorithm was used to study the imaging reconstruction of ERT ${ }^{[8][9]}$. But it has defects of premature and the small processing dimension, easy to reach the local optimal solution, and is difficult to achieve effective convergence with hundreds of split units. So it is difficult to completely meet the system requirements using one algorithm alone.

Based on the research and analysis above, a hybrid type imaging algorithm is proposed and discussed.

\section{The basic working principle of TERT}

Figure. 1 shows the simple working principle of TERT system. Under a certain current excitation, the change of the electrical conductivity in area $\Omega$ will cause the change of the measured boundary voltage $\mathrm{V}_{\mathrm{ij}}$. Thus by measuring the boundary voltages, the object (tuber) in the area $\Omega$ could be detected 


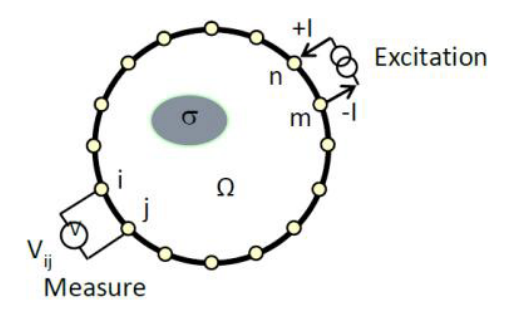

Figure 1. diagram of the TERT work.

The mathematic model of the forward problem of the TERT system is described as:

$$
\left\{\begin{array}{c}
\nabla \cdot(\sigma \cdot \nabla \varphi)=0 \\
\left.\sigma \frac{\partial \varphi}{\partial n}\right|_{\partial \Omega}=+I \\
\left.\sigma \frac{\partial \varphi}{\partial n}\right|_{\partial \Omega}=-I
\end{array}\right.
$$

where $I$ is injected current, $\sigma$ is the potential, $\left.\sigma \frac{\partial \varphi}{\partial n}\right|_{\partial n}$ is the derivative in the outward normal direction along the boundary, $\Omega$ is the whole target area.

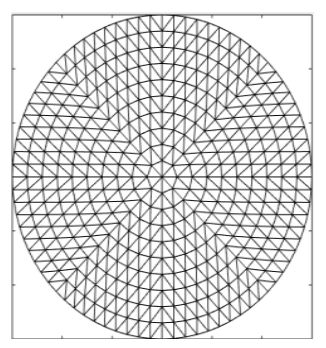

Figure 2. The pictorial diagram of the finite element model.

The finite element method is used to solve the forward problem in TERT system. The basic idea is to discrete the continuous solution domain into an assembly of finite elements. Such an assembly can better simulate or approximate the solution domain. If the mesh unit meets the convergence requirements, the approximate solution will converge to the exact FEM. The detailed steps including: 1) Determine of domain section parameters; 2) Selecting of field variable interpolation function in a unit; 3) Forming element characteristic matrix and element nodal equation; 4) Computing system matrix and integral equation; 5) Solving linear equations to get the field variable value.

Different topological types of finite element model will cause symmetry error and structural error ${ }^{[10]}$. Triangular element finite element method was used in the paper as shown in Fig. 2.

In Fig. 2, electrode number is 16, the simulation electrode is point electrode mode, the total number of nodes is 441 , the number of boundary nodes is 80 , the number of finite elements is 800 , field radius is $0.15 \mathrm{~m}$, the excitation current intensity is $30 \mathrm{~mA}$, the data acquisition using adjacent excitation mode.
In the TERT system, plant tuber is grown in soil, so the field can be approximated as two different kinds of material. By measuring, the set average resistivity of a tuber is about $6353 \Omega \cdot \mathrm{m}$ and the set average resistivity of the soil environment is about $1869 \Omega \cdot \mathrm{m}$

\section{Simulation research on image reconstruction algorithms of TERT}

The analysis result shows that the resistivity data obtained by MNR has obvious ladder and partition, and the number of units in the area is about 20. So the number of chromosomes in the GA algorithm is 30 , the maximum iteration number is 200, the crossover and mutation probability are 0.7 and 0.1 respectively.

Fitness function is:

$$
f=\frac{2}{\left\|V^{k}-V_{0}\right\|^{2}}
$$

In addition, in order to further measure the quality of the reconstructed image, the spatial image error SIE and space occupied area error E are introduced.

The spatial image error can represent the error of the position and the distribution of the reconstructed image:

$$
S I E=\frac{\sum_{i=1}^{M}\left|G_{S}^{*}(i)-G_{r}^{*}(i)\right|}{\sum_{i=1}^{M} G_{S}^{*}(i)}
$$

$G_{s}^{*}, G_{r}^{*}$ represent the set model and the reconstructed image respectively.

The space occupied area error can represent the relative value of the difference between the actual object area and the reconstructed image area is indicated.

$$
E=\frac{\left[S_{S}-S_{R}\right]}{S_{S}}
$$

TERT system is built to study the growth of plant tubers in the soil which are roughly round, so the simulation calculation is completed based on circular and ellipse. The simulation resistivity distribution is as follows:

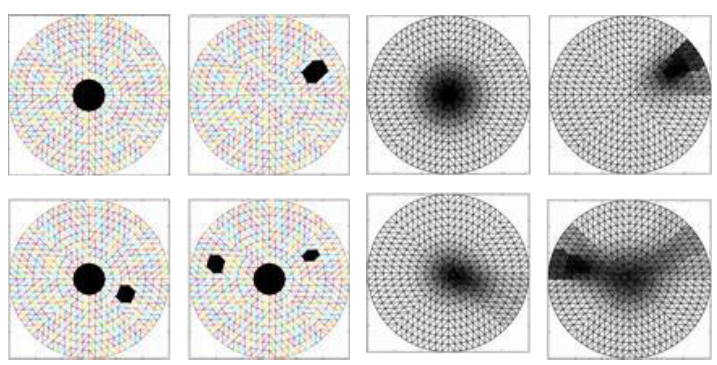




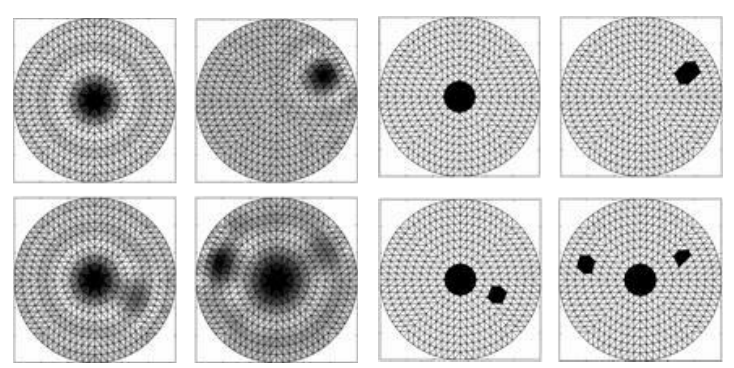

Figure 3. Image reconstruction results by using different algorithms.

Fig. 3 shows that the proposed algorithm LMG has obvious improvement. This algorithm can reconstruct clear field distribution patterns which are difficult with other two algorithms. Compared with the SIE parameters in Table.1, the hybrid algorithm is the most effective, which can improve the quality of the reconstructed image.

Table 1. Compare date results of different algorithms.

\begin{tabular}{|c|c|c|c|c|}
\hline \multirow{2}{*}{ Field } & Par & L & LM & LMG \\
\hline \multirow{2}{*}{$\mathrm{A}$} & SIE & 0.2483 & 0.1835 & 0 \\
& $E$ & 0.12 & 0.05 & 0 \\
\hline \multirow{2}{*}{$\mathrm{B}$} & SIE & 0.3648 & 0.2372 & 0 \\
& $E$ & 0.105 & 0.035 & 0 \\
\hline \multirow{2}{*}{$\mathrm{C}$} & SIE & 0.3852 & 0.3024 & 0 \\
& $E$ & 0.07875 & 0.06625 & 0 \\
\hline \multirow{2}{*}{$\mathrm{D}$} & $S I E$ & 0.04254 & 0.02879 & 0 \\
& $E$ & 0.1225 & 0.10375 & $1.25 \mathrm{e}-4$ \\
\hline
\end{tabular}

\section{Initial experimental results analysis}

and

In order to verify the practical application effect of the combination algorithm, the developed TERT system was used to obtain the actual experimental data. As shown in Figure 4, a composite array of electrodes is used in the system. The field diameter is about $300 \mathrm{~mm}$, the excitation frequency can be adjusted from $1 \mathrm{kHz}$ to $5 \mathrm{kHz}$, and the excitation current size can be adjusted from 0 to $30 \mathrm{~mA}$.

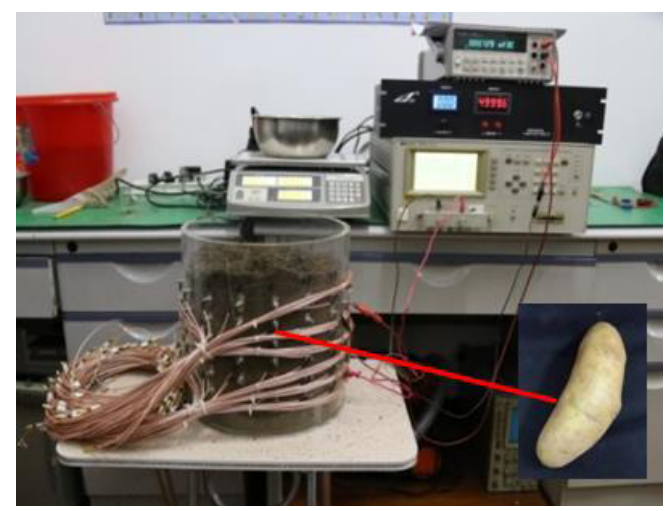

Figure 4. The developed TERT system with array electrodes.

In the actual experiment, the soil was collected in the farmland and adds water to saturation, the plant tuber (potato) was buried as shown in Fig. 5.
TERT system equidistant set up 6 layers of electrode array from top to bottom, spacing is $5 \mathrm{~cm}$. The tuber can only across the 2, 3, 4 layer electrode imaging area, therefore, only these three layers were imaged as follow:

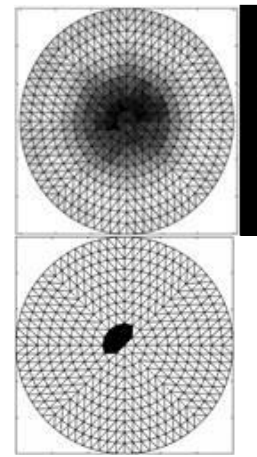

a) 2nd layer

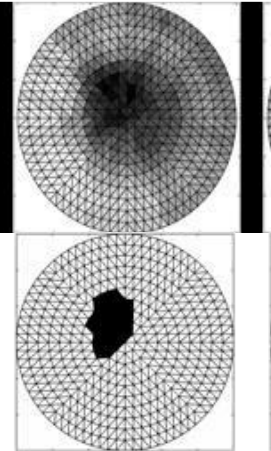

b) 3rd layer

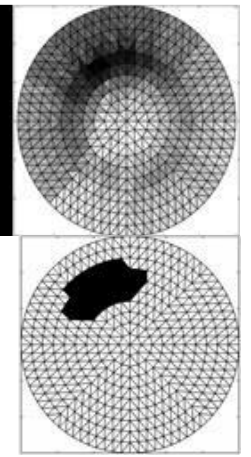

c) 4th layer
Figure 5. Image reconstruction results by using LM and LMG

In the comparison of the actual imaging parameters, the MNR evaluation parameter was used to represent the similarity between the calculated field and the actual field.

Figure 5 is image reconstruction results by using LM and LMG. Table 2 shows, in the actual experiment, due to the error of finite element model and the noise interference, the effect of hybrid algorithm is slightly worse, but still significantly outperforms the LBP and MNR algorithms, and can clearly reconstruct the potato profile.

Table 2. Actual imaging parameters.

\begin{tabular}{|c|c|c|c|}
\hline Field & Parameters & LM & LMG \\
\hline A & F & 2.1325 & 0.0352 \\
\hline B & F & 2.5864 & 0.00543 \\
\hline C & F & 3.7412 & 0.0624 \\
\hline
\end{tabular}

\section{Conclusion}

In this paper, linear back projection algorithm, modified Newton-Raphson algorithm and interval splitting were introduced to genetic algorithm. And a hybrid TERT image reconstruction algorithm was proposed which makes up for the deficiency of the single algorithm. It has effectively eliminated the transition region in the reconstructed image and achieved the transformation of imaging simulation from fuzzy to clear.

Experiments show that the hybrid algorithm has the following advantages: 1) this algorithm not only has a good effect on the field reconstruction of a single tuber, but also can reconstruct clear field distribution patterns which are difficult with other two algorithms. 2) it has effectively overcome the problem of poor local search ability of genetic algorithm, and improve the quality of the reconstructed image. 3 ) it has strong robustness. 


\section{Acknowledgement}

The authors would like to thank the National Natural Science Foundation of China (No.: 61271307).

\section{References}

1. Jian Ning, Yitian Sun, Qing Liu, Tianshi Ma, Hui Liu, Zhiming Wei. Development trend of intelligent precision agricultural equipment [J]. Mechanical and electrical products development and innovation. Volume 24(2), 2011.

2. Yanjun Zhang, Yu Chen, Deyun Chen. Sensitivity analysis and image reconstruction of electrical resistance tomography system [J]. Computer Science, 2010, 37 (8): 257-261.

3. Deyun Chen, Yanjun Zhang. An image reconstruction algorithm for electrical resistance tomography based on algebraic neural network [J]. Computer Engineering and application, 2009, 45 (32):19-21.

4. Liqing Xiao, Xiaogen Shao. Analysis and design of the finite element simulation model for electrical resistance tomography [J]. Journal of Chinese and instrument, 2008, 29 (2):354.360.

5. Peng, X. M.; Tan, W. X.; Wang, Y. P. Convolution back-projection imaging algorithm for downwardlooking sparse linear array three dimensional synthetic aperture radar[J]. PROGRESS IN ELECTROMAGNETICS RESEARCH-PIER. 2012, 129:287-313.

6. Liqing Xiao, Huaxiang Wang, Xiaoju $\mathrm{Xu}$ etc. Improved Newton - Raphson electrical resistance tomography image reconstruction algorithm $[\mathrm{J}]$. The Chinese Electrical Engineering Journal, 2012, 32 (1): 198-200. (8): 91-97.

7. Xia Bian, Liang Mi. Research Progress on genetic algorithm theory and Application [J]. Computer Application Research, 2010, 27 (7): 2425-2429.

8. Sanaz Asgarifar, Javad Frounchi. A novel two-stage genetic algorithm for image reconstruction of electrical resistance tomography $[\mathrm{J}]$. All Publications, 2012, 01(4).

9. Furman, A; Ferre, TPA; Warrick, AW. Optimization of ERT surveys for monitoring transient hydrological events using perturbation sensitivity and genetic algorithms [J].VADOSE ZONEJOURNAL, 2004, 3(2):1230-1239.

10. Liqing Xiao, Xiaogen Shao, Linlin Wang, et. Analysis and design of the finite element simulation model for electrical resistance tomography [J]. Journal of Chinese and instrument, 2008, 29 (2):354.360. 